\title{
Prostatakarzinom
}

\section{Kontinenz und Potenz nach radikaler Prostatektomie}

\section{Gibt es einen Zusammenhang zwischen Kontinenz und erektiler Potenz nach radikaler Prostatektomie? Dr. Marius Cristian Butea-Bocu, Kliniken Hartenstein, Bad Wildungen, stellte auf dem Deutschen Krebskongress hierzu Ergebnisse einer Untersuchung vor, welche dieser Frage nachging.}

In früheren Studien konnte gezeigt werden, dass ältere Patienten nach Prostatovesikulektomie einen größeren Urinverlust bis hin zur Inkontinenz zeigen als jüngere Patienten (1). Dies galt auch für den älteren Patienten mit nerverhaltender Operation. Insgesamt war der Urinverlust bei nicht nerverhaltend operierten Patienten aber höher als bei unilateral nerverhaltend operierten und bilateral nerverhaltend operierten Patienten (2). In der Anschlussheilbehandlung (AHB) wird in Bad Wildungen ein Kontinenztraining nach Otto et al. sowie ein physiotherapeutisches ED-Training angeboten.

In einem Zeitraum von 8 Jahren wurden bei allen Patienten, die bis zu 35 Tage postoperativ in der AHB waren, insbesondere 55 Parameter erfasst, insbesondere Funktionsparameter zu Beginn und Abschluss der AHB, onkologische Parameter aus den Arztberichten und personenbezogene Parameter. Es wurden die Daten von 20060 Patienten erhoben und statistisch ausgewertet, von denen 19355 (96,5\%) Angaben zur erektilen Funktion abgegeben hatten. Nach Abschluss der AHB war etwa ein Drittel der analysierten Patienten, kontinent. Von diesen lagen für $96 \%$ Angaben zur erektilen Funktion vor.

Im Ergebnis nahm der Anteil an Patienten mit Kontinenz im Alter ab, auch die erektile Potenz wurde geringer. Patienten $\leq 59$ Jahre waren in $46,9 \%$ der Fälle kontinent, in der Altersgruppe von 60-69 Jahren waren es 33,2\% der Patienten, und im Alter $\geq 70$ Jahre $24,3 \%$. In diesen Altersgruppen gaben $18,4 \%, 9,1 \%$ und $3,2 \%$ an, erektile Potenz zu besitzen. Im Rahmen der Frührehabilitation konnte bei etwa $12 \%$ der Patienten mit vollständiger Kontinenz eine erektile Funktion erreicht werden,

\section{Fazit}

Die Analyse an einer großen Patientenpopulation zeigt eine Korrelation zwischen Kontinenz und Potenz mit einer starken Altersabhängigkeit. Patienten unter 60 Jahren besitzen eine deutlich höhere Chance auf den Erhalt der Kontinenz sowie der Potenz.

so Butea-Bocu. Eine Korrelation zwischen Kontinenz und Potenz wurde über alle Altersgruppen signifikant gezeigt $(0,093 ; p<0,0001)$. Die Auswertung zeigte bei der Korrelation einen signifikanten Vorteil für die jüngeren Patienten.

Dr. Ine Schmale, Westerburg

\section{Literatur}

1. Borrusch $\mathrm{H}$ et al. Harninkontinenz nach radikaler Prostatavesikulektomie. Haben Patienten ein erhöhtes Risiko? Der Urologe 2011; 50: 457-461.

2. Borrusch et al. Wert und Risiko der Erhaltung der neurovaskulären Bündel bei radikaler Prostatovesikulektomie. DGU 2011, Abstract P15.

Quelle: AUO-Symposium „Grenzen und Chancen der Therapie beim Prostatakarzinom" im Rahmen des Deutschen Krebskongresses (DKK) am 21. Februar 2018, Berlin. 